\title{
Degradación de Efluentes Líquidos Mediante Lechos Fluidizados
}

\author{
Agustín F. Navarro* y Luis A. Palladino \\ Universidad Nacional de La Plata, Facultad de Ingeniería, Departamento de Ingeniería Química, \\ Calle 48 y 115, 1900 La Plata-Argentina (e-mail: anavarro@ing.unlp.edu.ar)
}

*autor a quien se debe ser dirigida la correspondencia

\begin{abstract}
Resumen
El objetivo del trabajo es determinar las eficiencias de degradación de la materia orgánica de un efluente líquido, mediante un reactor biológico de lecho fluidizado. Se determina también los límites de carga orgánica y caudal para el equipo experimental. El material soporte utilizado es carbón activado con un diámetro medio de $1 \mathrm{~mm}$, ocupando en condición de lecho fijo un $38 \%$ del total de la columna líquida. Se operó con caudales de alimentación comprendidos entre 20 y $300 \mathrm{ml} / \mathrm{min}$ y reciclos entre 1400 y $4800 \mathrm{ml} / \mathrm{min}$. La carga orgánica de ingreso se varió desde 74 a 571 mg/l de DQO y se obtuvo eficiencia de degradación del orden de $89 \%$. Se observa una buena eficiencia de reducción de la materia orgánica respecto a otros sistemas de tratamiento biológico, pero debido al constate crecimiento bacteriano requiere ajustes durante la operación.
\end{abstract}

Palabras clave: lechos fluidizados, efluentes líquidos, tratamiento biológico, materia orgánica

\section{Wastewater Degradation using Fluidized Beds}

\begin{abstract}
The purpose of this study is deterring efficiencies for organic matter degradation from wastewaters using a biological fluidized bed reactor. Additionally, the organic load limits and volume flow rate for the experimental setup were determined. Backfill material is activated coal with a mean diameter of 1 $\mathrm{mm}$ filling $38 \%$ of the total liquid column in fixed bed condition. The reactor was operated with an effluent volume flow rate between 20 and $300 \mathrm{ml} / \mathrm{min}$ and recycles between 1400 and $4800 \mathrm{ml} / \mathrm{min}$. The input organic load varied from 74 to $571 \mathrm{mg} / \mathrm{l}$ as COD and degradation efficiency of the order of $89 \%$ was obtained. It is observed that the system presents good efficiency of the organic matter oxidation with respect to other systems for biological treatment. However, due to the constant bacterial growth, the system requires adjustment during operation.
\end{abstract}

Keywords: fluidized beds, wastewater, biological treatment, organic matter 


\section{INTRODUCCIÓN}

Los efluentes líquidos provenientes de descargas urbanas e industriales que presentan una alta carga orgánica medida a través de la Demanda Bioquímica de Oxígeno (DBO) o Demanda Química de Oxígeno (DQO) deben ser tratados para permitir su vuelco a los cuerpos de agua superficial cumpliendo con los estándares de calidad correspondientes. Existen diversos métodos de tratamiento o depuración que permiten disminuir notablemente la DBO o DQO final del efluente. Entre los procedimientos mas tradicionales están los sistemas de lodos activados (biomasa en suspensión) y biodiscos, lechos percoladores y fluidizados (biomasa en películas fija). En este último caso, se utiliza un lecho granular de partículas acondicionadas dentro de una columna que tiene incorporados en su parte inferior entradas de efluente a tratar y reciclo, y en su parte superior salida del efluente tratado. Los caudales de alimentación y reciclo tienen que ser tales que sustenten el lecho de partículas promoviendo su fluidización (Yang, 2003) y la degradación de la materia orgánica a través de los microorganismos adosados a la superficie de las partículas (Shieh et al., 1981; Nicollela et al., 2000; Wang et al., 2007). La inyección de aire u oxígeno es imprescindible para lograr una buena aireación del sistema, se puede incorporar en la corriente de ingreso o dentro del propio lecho (Rajasimman y Karthikeyan, 2007). A la salida del efluente tratado se suele colocar un tanque de sedimentación o clarificación del mismo.

Normalmente en la literatura a los reactores de lecho fluidizado que utilizan, como en este trabajo, carbón activado como soporte, se los conoce como reactores GAC (Granular Activated Carbon) basados en BFB (Biological Fluidized Bed). La metodología consiste en pasar el efluente hacia arriba atravesando el lecho en condiciones más allá del punto en el cual las fuerzas de arrastre sobre las partículas igualan las fuerzas gravitatorias, manteniendo el estado de fluidización particulada (Sutton y Mishra, 1994). En este tipo de sistemas se puede trabajar a una muy alta concentración de biomasa por la gran superficie del medio soporte, logrando de este modo buena reducción de la materia orgánica (Nicollela et al., 2000). Otras partículas comunes que son utilizadas en la práctica son arena, arcilla y carbón, en general de pequeña granulometría para favorecer su sustentación por medio del efluente. Es importante mantener un nivel de biomasa constante por lo que el excedente de la misma se puede eliminar ya sea permitiendo el roce entre partículas y partículas y paredes del recipiente favoreciendo el desprendimiento del exceso, o rebose continuo / periódico de las partículas fuera del lecho y hacia un dispositivo donde la biomasa se separa de las partículas y éstas son incorporadas nuevamente al lecho, con generación de barros (Perry y Green, 1999).

El objetivo del presente estudio es encontrar las eficiencias de degradación de la materia orgánica contenida en un efluente líquido sintetizado en laboratorio frente a distintos caudales de alimentación y carga orgánica asociada. Del mismo modo obtener valores límites de esos parámetros que hagan posible una operación estable y eficiente del reactor.

\section{METODOLOGIA}

El modelo a escala piloto fue construido en laboratorio y consta de una columna de acrílico de $10 \mathrm{~cm}$ de diámetro y $1,10 \mathrm{~m}$ de altura, con una salida lateral ubicada a $20 \mathrm{~cm}$ por debajo del tope de $2,5 \mathrm{~cm}$ de diámetro (Figura 1). El soporte de relleno está compuesto por una malla de acero inoxidable colocada a $10 \mathrm{~cm}$ de la base, por debajo del mismo se alimenta el efluente a tratar y el reciclo.

Una pileta lateral de $15 \mathrm{~cm}$ por $15 \mathrm{~cm}$ de base y una altura de $20 \mathrm{~cm}$ contiene un vertedero en la parte superior para descarga del efluente tratado y una salida en el fondo para el reciclo. En el interior de la pileta se ha incorporado un burbujeador de aire con caudales comprendidos entre 1,5 I/min y 2,5 I/min para mantener condiciones aeróbicas en la misma. El material soporte utilizado es carbón activado de pequeña granulometría (diámetro equivalente de $1 \mathrm{~mm}$ aproximadamente), que se cargó en la columna de manera tal de alcanzar una altura de lecho fijo de $30 \mathrm{~cm}$, cuya función es la de contar con una gran superficie expuesta para la adhesión de microorganismos.

La altura del lecho se estableció de tal forma que quedara un volumen considerable por encima del mismo, ocupado por la masa de líquido tratada, permitiendo de esta forma trabajar con distintas velocidades de fluidización y por ende distintas expansiones de lecho. Completan el sistema dos 
bombas: de alimentación (peristáltica) y reciclo (regenerativa) y un tanque de 1000 I de capacidad conteniendo el efluente a tratar.

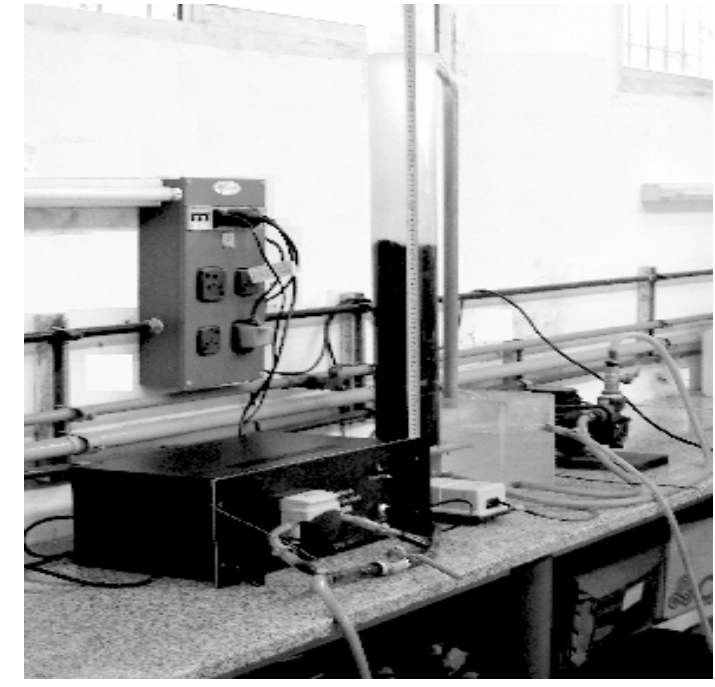

Fig. 1: Modelo a escala piloto

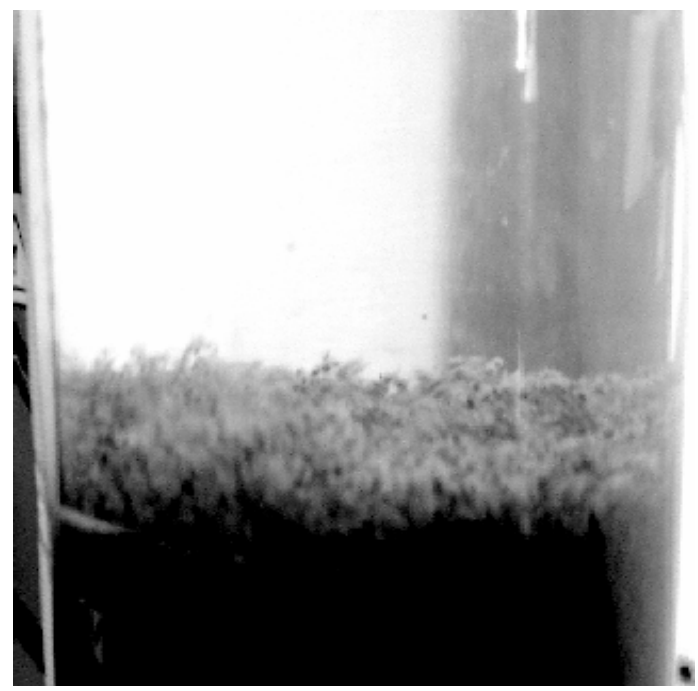

Fig. 2: Partículas fluidizadas en interfase

En la Figura 2 se puede observar la fluidización de partículas y biomasa adherida en su superficie, correspondiente a la interfase lecho fluidizado - efluente tratado.

En el inicio de la operación se mantuvieron condiciones de mínima fluidización (altura de lecho de 37 $\mathrm{cm}$ ) para favorecer el desarrollo de microorganismos adosados al medio soporte y con caudal de recirculación exclusivamente. Se agregaron aproximadamente $10 \mathrm{~g} / \mathrm{d}$ de la base con que se elaboró el efluente. Luego de aproximadamente cuatro semanas se observó crecimiento bacteriano visible. A partir de este momento se realizaron ensayos con caudales de alimentación comprendidos entre 20 $\mathrm{ml} / \mathrm{min}$ y $300 \mathrm{ml} / \mathrm{min}$ y concentraciones de carga orgánica, medida como DQO, variando desde 74 $\mathrm{mg} / \mathrm{l}$ y hasta $571 \mathrm{mg} / \mathrm{l}$. En condición de operación normal el caudal de reciclo mantuvo valores comprendidos entre $1400 \mathrm{ml} / \mathrm{min}$ y $4800 \mathrm{ml} / \mathrm{min}$ correspondiente a velocidades de fluidización de 0,3 $\mathrm{cm} / \mathrm{s}$ y $1,01 \mathrm{~cm} / \mathrm{s}$, proporcionando una altura de lecho fluidizado entre $40 \mathrm{~cm}$ y $60 \mathrm{~cm}$. Debido al crecimiento de la biomasa sobre el medio soporte se disminuyó diariamente la velocidad de fluidización para mantener la operación estable. Adicionalmente se realizaron purgas semanales del exceso de biomasa y parada total con lavado completo del sistema al final de cada serie de ensayos.

El efluente a tratar fue sintetizado en laboratorio mediante la dilución de un sustituto lácteo (ya utilizado con éxito en otros sistemas de tratamiento) hasta lograr la DQO con la que se iba a trabajar en las pruebas. La determinación de este parámetro se realizó mediante un Equipo marca $\mathrm{HACH}$ compuesto por Reactor modelo 45600 y Espectrofotómetro DR-2000, Hach Corp, Loveland (USA).

\section{RESULTADOS Y DISCUSION}

Se realizaron un total de 24 ensayos en diferentes épocas del año. Se midieron distintos parámetros: Carga orgánica en el efluente de entrada (DQO Ent) y de salida (DQO Sal), Caudal de ingreso (q), Temperatura en la pileta de reciclo (T), Oxígeno Disuelto en pileta (ODP) y en lecho (ODT), pH en pileta, Longitud de lecho fluidizado (Lf). Se calculó Eficiencia de degradación ( $\eta$ ). La Tabla 1 muestra los resultados obtenidos.

Las primeras ocho experiencias fueron exploratorias tendientes a determinar el comportamiento del sistema (Navarro y Palladino, 2006) y las siguientes tratando de encontrar zonas de máximas eficiencias. Las mediciones fueron realizadas cuando la longitud del lecho fluidizado estuvo comprendida entre $40 \mathrm{~cm}$ y $60 \mathrm{~cm}$, por debajo de $40 \mathrm{~cm}$ el lecho se compacta y por encima de $60 \mathrm{~cm}$ entra en la zona de inestabilidad con arrastre de biomasa. 
Tabla 1: Parámetros medidos durante la operación del lecho fluidizado

\begin{tabular}{|c|c|c|c|c|c|c|c|c|}
\hline $\begin{array}{c}N^{\circ} \text { de } \\
\text { Ensayo }\end{array}$ & $\begin{array}{c}\text { DQO Ent } \\
\mathrm{mg} / \mathrm{l}\end{array}$ & $\begin{array}{c}\text { DQO Sal } \\
\mathrm{mg} / \mathrm{l}\end{array}$ & $\begin{array}{c}\mathrm{q} \\
\mathrm{ml} / \mathrm{min}\end{array}$ & $\begin{array}{c}\mathrm{T} \\
{ }^{\circ} \mathrm{C}\end{array}$ & $\begin{array}{c}\mathrm{OD}(\mathrm{P} / \mathrm{T}) \\
\mathrm{mg} / \mathrm{l}\end{array}$ & $\mathrm{pH}$ & $\begin{array}{c}\mathrm{Lf} \\
\mathrm{cm}\end{array}$ & $\begin{array}{c}\eta \\
\%\end{array}$ \\
\hline 1 & 100 & 48 & 90 & 32 & - & 7,4 & 64 & 52 \\
\hline 2 & 172 & 102 & 160 & 31 & - & 6,8 & 64 & 41 \\
\hline 3 & 149 & 90 & 120 & 38 & - & 7,4 & 50 & 40 \\
\hline 4 & 144 & 57 & 120 & 41 & - & 7,6 & 55 & 60 \\
\hline 5 & 206 & 140 & 70 & 35 & - & 6,8 & 43 & 32 \\
\hline 6 & 106 & 70 & 120 & 35 & - & 7,4 & 49 & 34 \\
\hline 7 & 109 & 69 & 120 & 32 & - & 7,4 & 56 & 37 \\
\hline 8 & 194 & 134 & 90 & 29 & - & 7,4 & 53 & 32 \\
\hline 9 & 216 & 147 & 25 & - & - & - & 40 & 32 \\
\hline 10 & 1453 & 669 & 170 & - & - & - & 67 & 54 \\
\hline 11 & 290 & 216 & 100 & 32 & $5,0-3,8$ & 7,1 & 43 & 26 \\
\hline 12 & 440 & 347 & 120 & 37 & $5,5-0,5$ & 7,1 & 38 & 21 \\
\hline 13 & 571 & 299 & 300 & 30 & $7,0-4,5$ & 7,1 & 49 & 48 \\
\hline 14 & 196 & 134 & 90 & 30 & $6,5-4,5$ & 7,1 & 50 & 29 \\
\hline 15 & 99 & 33 & 40 & 36 & $12-6,0$ & 7,1 & 40 & 67 \\
\hline 16 & 295 & 38 & 40 & 43 & $7,0-2,0$ & 7,1 & 45 & 87 \\
\hline 17 & 79 & 31 & 60 & 46 & $8,0-5,0$ & 7,1 & 46 & 61 \\
\hline 18 & 274 & 36 & 60 & 36 & $4,5-2,0$ & 7,1 & 58 & 87 \\
\hline 19 & 118 & 32 & 20 & 42 & $7,5-2,4$ & 7,1 & 43 & 73 \\
\hline 20 & 278 & 31 & 20 & 42 & $5,6-3,8$ & 7,1 & 41 & 89 \\
\hline 21 & 74 & 31 & 80 & 34 & $6,0-4,6$ & 7,1 & 43 & 58 \\
\hline 22 & 240 & 65 & 80 & 31 & $4,0-2,3$ & 7,1 & 70 & 73 \\
\hline 23 & 72 & 32 & 100 & 36 & $7,0-5,3$ & 7,1 & 48 & 56 \\
\hline 24 & 310 & 78 & 100 & 38 & $3,2-0,6$ & 7,1 & 58 & 75 \\
\hline
\end{tabular}

Si se correlaciona la carga orgánica de ingreso (COI) calculada como DQO Ent por el Caudal de ingreso y por unidad de sección transversal vs. Eficiencia de degradación para las diferentes experiencias, se encuentra que en general a menor carga orgánica de entrada existe una mayor eficiencia de degradación y viceversa (Figura 3). Se ha trazado una línea de tendencia de los puntos obtenidos que así lo indica. No se ha incluido el punto de máxima carga correspondiente a la experiencia 10, el cual muestra una notable eficiencia de degradación.

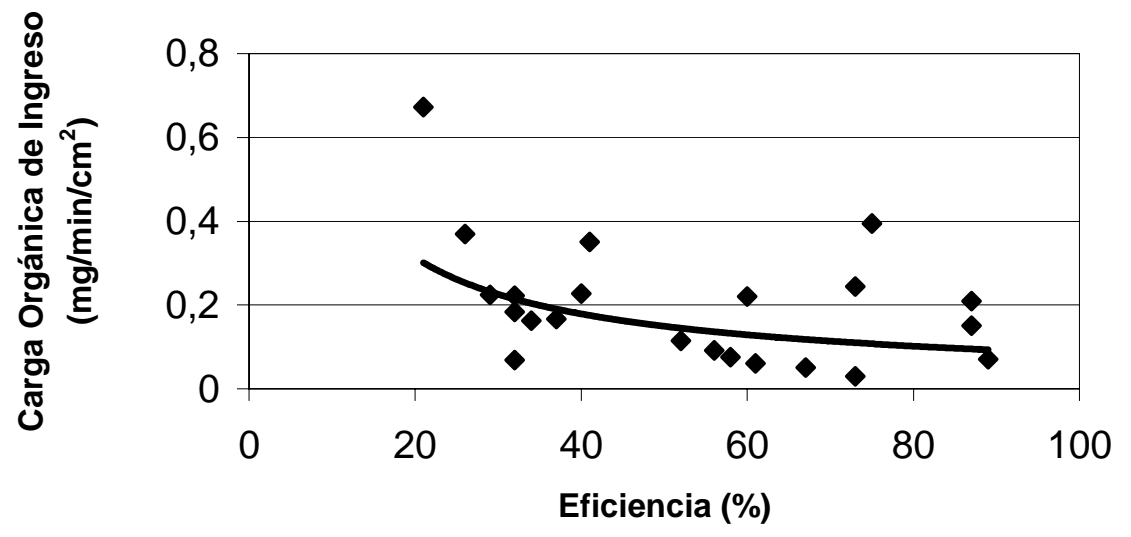

Fig. 3: Eficiencia de Degradación en función del ingreso de materia orgánica

En cuanto a la parte operativa, trabajar con recirculación exclusivamente tiene el inconveniente de un aumento de temperatura en todo el sistema debido al aporte calórico de las pérdidas friccionales dentro de la bomba de reciclo, lo cual origina evaporación del líquido en la columna que es necesario reponer regularmente. En el caso de aporte de aire, se encontró que un caudal de 1,5 I/min generaba zonas anaeróbicas dentro del lecho y aumentando dicho caudal a 2,5 l/min se lograba una operación 
completamente aeróbica. En la etapa de operación normal con los distintos caudales ensayados se observa con el transcurso del tiempo un aumento de la altura del lecho fluidizado de manera sostenida, debido al crecimiento bacteriano adosado a la superficie soporte, lo cual hace que se expanda mas allá de la condición inicial de trabajo. La altura fue ajustada diariamente disminuyendo el valor del caudal de reciclo o lo que es lo mismo la velocidad de fluidización. En el caso de los mayores caudales, para llevar el lecho a condiciones normales de operación se realizó una eliminación de la biomasa en exceso. Esto se puede hacer de dos formas: a) Trabajar con una velocidad de fluidización tal que se permita la atrición entre partículas cargadas con biomasa y entre partículas y las paredes del reactor a fin de que los sólidos biológicos sean arrastrados fuera del lecho y salgan con el efluente, un clarificador será el encargado de separar la biomasa del efluente tratado. b) Hacer que las partículas rebosen de manera continua o periódica fuera del lecho, luego de lo cual mediante algún mecanismo como agitación, hidrociclón, etc. se separa la biomasa de las partículas, regresando éstas al lecho fluidizado. En nuestro caso se probó la incorporación de pulsos (velocidades de fluidización altas e instantáneas) que permitieran un desprendimiento de la biomasa del material soporte sin pérdida del mismo (Figura 4). El procedimiento consistió en lo siguiente:

1. Apertura total de la válvula de recirculación hasta la expansión completa del lecho.

2. Cierre completo de dicha válvula e interrupción de la operación de la bomba de recirculación.

3. Mayor apertura de la válvula de alimentación hasta lograr una fluidización mínima del lecho.

4. Mantenimiento de este régimen de operación hasta obtener arrastre del exceso de la biomasa hacia la pileta.

5. Incorporación de una corriente secundaria de agua pura en la pileta que aumente la eficiencia de remoción de biomasa fuera de la misma.

6. Inicio de operación normal.

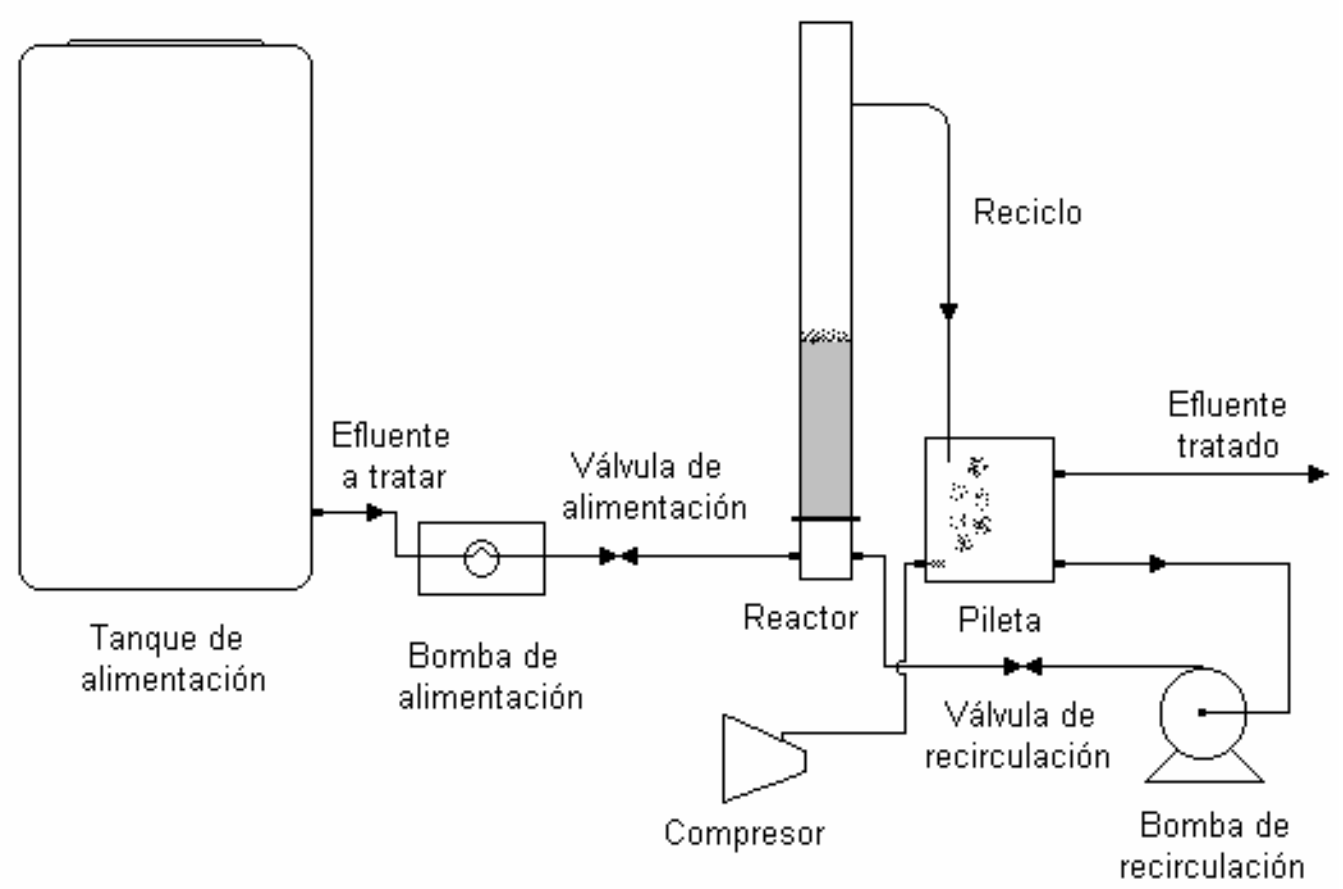

Fig. 4: Esquema de la Planta de Tratamiento por Lecho Fluidizado

Los caudales de reciclo para este sistemas están en el orden de 12 a 200 veces el caudal de alimentación, muy superiores a los utilizados en lechos de escurrimiento, lo cual resulta en una variable importante para la operación estable del sistema. Las temperaturas en la pileta siempre fueron elevadas en cualquier época en que se realizaron los ensayos debido a la recirculación del efluente. Las condiciones de $\mathrm{pH}$ permanecieron neutras durante todas las experiencias. El lecho siempre mantuvo condiciones aeróbicas salvo en dos oportunidades en que era alta la carga orgánica de ingreso sumado a un notable desarrollo bacteriano. Las velocidades de fluidización empleadas aquí, $0,30 \mathrm{~cm} / \mathrm{s}$ a $1,01 \mathrm{~cm} / \mathrm{s}$, son similares a las utilizadas por otros autores (Sarrouh et 
al., 2008). La expansión del lecho durante la operación alcanza valores del 50\%, llegando a órdenes del $70 \%$ con un crecimiento excesivo de la biomasa presente. La eficiencia máxima lograda fue del $89 \%$ comparable con la obtenida por otros autores (Rajasimman y Karthikeyan, 2007) aunque trabajando en nuestro caso con cargas orgánicas mucho menores.

\section{CONCLUSIONES}

Se ha probado un sistema de lecho fluidizado para el tratamiento de efluentes líquidos con una determinada carga orgánica, mostrando un comportamiento adecuado a los fines buscados principalmente en la zona de bajos caudales. Se obtuvieron resultados de la degradación de la materia orgánica como así también de parte de las características hidrodinámicas del lecho.

Se observa que es necesario un estricto control diario del lecho, principalmente en lo que se refiere a una buena oxigenación del mismo tendiente a impedir la aparición de zonas anaeróbicas. El crecimiento constante de la biomasa es un factor a tener en cuenta, ya que la misma hace elevar la interfase lecho - efluente tratado de manera sostenida en el tiempo, con el riesgo de sobrepasar el límite superior de trabajo.

Para concluir, operando con caudales de alimentación entre $20 \mathrm{ml} / \mathrm{min}$ y $300 \mathrm{ml} / \mathrm{min}$ y de reciclo entre $1400 \mathrm{ml} / \mathrm{min}$ y $4800 \mathrm{ml} / \mathrm{min}$ (velocidades de fluidización de $0,30 \mathrm{~cm} / \mathrm{s}$ y $1,01 \mathrm{~cm} / \mathrm{s}$ ) y cargas orgánicas de entrada comprendidas entre $74 \mathrm{mg} / \mathrm{l}$ y $571 \mathrm{mg} / \mathrm{l}$, se logran eficiencias de reducción de la materia orgánica comprendidas entre $21 \%$ y $89 \%$. Las eficiencias mayores corresponden a bajos caudales y máximas cargas orgánicas.

\section{REFERENCIAS}

Navarro, A. F. y L. A. Palladito; Degradación de Efluentes Líquidos mediante Lechos Fluidizados, Actas del XXII Interamerican Congress of Chemical Engineering, 11b Pollution Remediation (106032). Buenos Aires, Argentina 1 al 4 de Octubre (2006).

Nicolella, C., M.C.M. van Loosdrecht y J.J. Heijnen; Wastewater Treatment whit Particulate Biofilm Reactor, Journal of Biotechnology: 80, 1-33 (2000).

Perry R.H. y D. Green: Perry's Chemical Engineering Handbook, $7^{\text {th }}$ edición, Sec. 25, 75 - 76, Mc Graw Hill Companies, Inc. United State of America (1999).

Rajasimman, M. y C. Karthikeyan; Aerobic Digestion of Starch Wastewater in a Fluidized Bed Bioreactor with Low Density Biomass Support, Journal of Hazardous Materials: 143, 82-86 (2007).

Sarrouh, B.F., A. Converti y S. S. da Silva: Evaluation of Hidrodynamic Parameters of a Fluidized Bed Reactor with Immobilized Yeast, J. Chem. Technol. Biotechnol: 83, 576-580 (2008).

Shieh, W.K., P.M. Sutton y P. Kos; Predicting Reactor Biomass Concentration in a Fluidized Bed System, Journal WPCF: 53 (11), 1574-1684 (1981).

Sutton, P.M. y P.N. Mishra; Activated Carbon Based Biological Fluidized Beds for Contaminated Water and Wastewater Treatment: A State-of-Art Review, Water Science and Technology: 29 (10-11), 309 -317 (1994).

Wang, X.C., H.L. Yuan, Y.J. Liu y P.K. Jin; Fluidized Pellet Bed Birreactor; a Promising Technology for Onsite Wastewater Traetment and Reuse, Water Science and Technology: 55, 59-67 (2007).

Yang, W.C.; Handbook of Fluidization and Fluid Particle System, Ed. by. CRC Press. N.Y. (2003). 